\title{
El conocimiento profesional de los (as) profesores (as) de ciencias: algunos aspectos centrales en el desarrollo de la línea de investigación ${ }^{1}$ \\ The professional knowledge of (as) the professors (as) of sciences: some central aspects in the development of the line of investigation
}

Carmen Alicia Martínez Rivera

\section{Resumen}

Se presenta una revisión desde la cual es posible señalar al conocimiento profesional de los (as) profesores(as) de ciencias, como un problema de gran relevancia en la didáctica de las ciencias. Se abordan antecedentes importantes en el surgimiento de esta línea de investigación.

\section{Palabras clave:}

Didáctica de las ciencias, conocimiento profesional, profesores(as) de ciencias, pensamiento del(a) profesor(a).

\section{Abstract}

A revision appears from which it is possible to indicate to the Professional Knowledge of (ace) the Profesores (as) de Cadencies, like a problem of great relevance in the Didactics of Sciences. Excellent antecedents in the sprouting of this line of investigation are approached.

\section{Key words:}

Didactics of sciences, professional knowledge, profesores(as) of sciences, thought del(a) profesor(a).

\section{Introducción}

"[...] Y le pedí a Dios que si salvaba mi vida y la de mis hijos dejaría este trabajo [prostituta] y... me volvería maestra”. Se salvaron de la avalancha y ella se volvió madre comunitaria. Relato de Molina (2006), sobre la tragedia de Armero en 1985.

1 Elaborado en el marco del proyecto de investigación "El conocimiento profesional de los profesores de ciencias de primaria sobre el conocimiento escolar en el Distrito Capital”. Colciencias-Universidad Distrital Francisco José de Caldas-Universidad Pedagógica Nacional. Y con base en C. Martínez (2008). El conocimiento profesional de los profesores de ciencias (CPPC). Desarrollos de la línea de investigación. Documento preparado para el seminario doctoral "El conocimiento profesional de los profesores de ciencias (CPPC)", ofrecido en la Universidad Distrital Francisco José de Caldas. Abril de 2008. Doctorado Interinstitucional en Educación.

2 Profesora de la Universidad Distrital Francisco José de Caldas. camartinezr@udistrital.edu.co 
La anterior petición es una mirada que muchas personas tienen respecto a lo que significa ser maestro: sacrificio, servicio, vocación. En este caso, la mujer ofrece como sacrificio el ser maestra, a cambio de no perecer en la terrible avalancha. ${ }^{3}$ Justamente, en este sentido el libro de Freire, Cartas a quien pretende enseñar, realiza una crítica al apelativo que se usó en Brasil para denominar al maestro o profesor de escuela, el de "tía"; y como lo indica Torres (1994), es una trampa mortal para la profesión del (a) profesor (a), con la que se supone se busca "ensalzar el ego del docente", con términos como: apóstol, héroe, jardinero, entre otros.

A los anteriores papeles que se les han asignado a los (as) profesores (as) cabría agregar el de "segundos padres o madres", manteniendo la misma trampa mortal. El cuestionamiento, entonces, que nos generan estas visiones es el de querer saber cuál es el papel del (a) profesor (a) como profesional. ¿Qué significa que se asuma como tal?, y ¿ cómo se ha venido desarrollando esta perspectiva? Estos son algunos de los aspectos que desarrollaremos en este escrito.

\section{Primeras aproximaciones a la línea de investigación}

Al revisar los primeros Handbook vemos cómo tienden a mostrar la educación del (a) profesor (a), implícita o explícitamente, como un asunto no problemático, como lo afirman Munby y Russell (1998); sin embargo, señalan estos mismos autores que en la última década, un incremento en el flujo de Handbooks en varias áreas de investigación educativa testifica el crecimiento del conocimiento de los(as) profesores(as). En este mismo sentido, y en particular, a cerca de la investigación sobre el pensamiento del (a) profesor (a), nos indican Clark y Peterson que "El Second Handbook of Research on Teaching (Travers, 1973) no incluía ningún capítulo sobre la investigación de los procesos de pensamiento de los docentes; en rigor, ni siquiera la mencionaba" (1997, p. 528).

El escrito de Clark y Peterson (1997, p. 443) señala claramente que los trabajos en torno al pensamiento,

3 Esta frase la escribo compartiendo el gran dolor de mis amigos.

REVISTA CIENTÍFICA / ENERO -DICIEMBRE DE 2009 / No. 11 / BOGOTÁ, D.C. planificación y toma de decisiones de los docentes se ubican en un contexto psicológico de la enseñanza, y parten de la consideración de que los procesos de pensamiento influyen decisivamente en la conducta “[...] e incluso la determinan". Se resalta que las primeras investigaciones sobre el conocimiento de la materia de los(as) profesores (as), está en el marco de la denominada investigación "proceso- producto" (Dunkin y Biddle, 1974), que busca encontrar relaciones estadísticas entre lo que los (as) profesores (as) saben y el logro de los estudiantes, incluso hoy se ha abandonado la idea de que quienes más saben sobre el contenido son quienes lo pueden enseñar (Grossman et ál., 2005).

Clark y Peterson (1997) ubican el trabajo de Jackson (1968), como uno de los primeros que intentó comprender y describir los constructos y procesos mentales que guían la conducta de los (as) profesores (as), en su obra La vida en las aulas. Estos autores señalan que el trabajo de Jackson no asumía las maneras de investigar del momento: métodos correlacionales y experimentales. No se entendía cómo la descripción de actividades realizadas en el aula podría aportar en la eficacia de la enseñanza. De tal modo que podríamos decir que una de las primeras dificultades en el desarrollo de la línea fue la concepción de investigación, que no correspondía a la aceptada en ese momento.

Parece que fue decisivo en el reconocimiento de la investigación sobre el pensamiento del (a) profesor (a), como un nuevo programa de investigación, el trabajo realizado en junio de 1974, en el que el National Institute of Education convocó a la Conferencia Nacional de Estudios sobre la Enseñanza. En el marco de este evento, la sexta comisión sobre "la enseñanza como tratamiento clínico de la información", presidida por Lee Shulman, fundamenta y define el programa de investigación sobre procesos de pensamiento de los docentes (Clark y Peterson, 1997). Esta comisión también señaló la necesidad de entender y conocer los procesos psicológicos de los (as) profesores (as), para poder comprender e incidir en lo que ellos hacen.

La concepción docente que asumió esta comisión fue relacionada con la profesión médica, señalan 
Clark y Peterson, que así, la sexta comisión presentó una imagen del docente como profesional, y promovió de manera indirecta la creación de un instituto de investigación de la enseñanza en la Universidad del estado de Michigan en 1976, que fue el primero en emprender un amplio programa de investigación sobre procesos de pensamiento de maestros.

Fijémonos que en este proceso, dos elementos se pueden resaltar como relevantes: la validación de la comunidad al destacar la "existencia" de un problema particular relacionado con el profesor, inicialmente de tipo más psicológico y conductual, en el que influyeron las consideraciones respecto a cómo se investiga, de ahí las dificultades del trabajo de Jackson (1968), pues lo que pareciera ser una perspectiva de tipo metodológico, seguramente estaba imbricada en una perspectiva epistemológica. El otro elemento relevante tiene que ver con la incidencia de una perspectiva conceptual, desde la cual se asume al profesor como profesional, en el paralelo realizado con el médico; según la propuesta elaborada con la dirección de Shulman en 1974, y desde la cual el profesor produce conocimiento al integrar diferentes fuentes de información, tanto del contexto como de la investigación en educación; construye sus propuestas de trabajo en atención a esas fuentes, pero también a sus propias expectativas, creencias y propósitos; revisa y reflexiona permanentemente sobre sus decisiones pasadas, y vuelve a empezar.

En particular, Shulman (2001: 174) indica que los (as) profesores (as) elaboran los siguientes conocimientos: conocimiento de la materia impartida; conocimientos pedagógicos generales (manejo y organización de la clase, que trascienden el ámbito de la asignatura); conocimiento del currículo (materiales y programas); conocimiento pedagógico del contenido ("esa especial amalgama entre materia y pedagogía que constituye una esfera exclusiva de los maestros, su propia forma especial de comprensión profesional"); conocimiento de los educandos y de sus características; conocimiento de los contextos educacionales. Incluso elabora una propuesta de modelo de razonamiento y ac-

4 En la traducción revisada se alude a "conocimiento pedagógico de la materia”. ción pedagógicas, de tal manera que aborda una aproximación a la caracterización del proceso de construcción de este conocimiento.

Es interesante anotar que esta motivación, por lo que significa ser profesional, también se evidenció en otras disciplinas, en el coloquio sobre educación profesional realizado en 1972, en Massachussets, aunque en una perspectiva más general, tal como lo indica Schön (1996). En este se tomó conciencia sobre las situaciones de complejidad e incertidumbre de los problemas de las profesiones.

Además, el interés por esta línea de trabajo no ha tenido una única perspectiva, tal como lo observamos en el cuadro 1, en la que recogemos algunos de los trabajos señalados por Gallego (1991) en el desarrollo de la línea de investigación sobre el pensamiento del (a) profesor (a) en aproximación al estudio de las "teorías y creencias de los profesores".

Indica Gallego (1991, p. 288) que los diferentes estudios señalan que los trabajos sobre el pensamiento del (a) profesor (a), han pasado hacia posiciones menos racionalistas y más antropológicas y filosóficas. Se ha dado un cambio de la investigación, pasando de la presentación de modelos en la toma de decisiones hacia el pensamiento en acción "y al estudio del Conocimiento práctico del profesor".

En este sentido, la revisión realizada por Clark y Peterson (1997) analiza las investigaciones y el desarrollo de la línea de procesos de pensamiento de los docentes con base en el modelo propuesto por ellos, llamado "modelo del pensamiento y la acción del maestro". Este se centra en dos grandes dominios que ellos señalan como procesos de pensamiento y acciones de los maestros y sus efectos observables. De tal modo que una diferencia fundamental parece ser el si son o no observables. "Los procesos de pensamiento de los docentes ocurren 'en la cabeza de los docentes' y por lo tanto no son observables. En cambio, la conducta del docente, la del alumno y puntuaciones que califican el rendimiento del alumno, son fenómenos observables" (Clark y Peterson, 1997, p. 448). Estos dominios, además, se diferencian por el enfoque 


\begin{tabular}{|c|c|c|}
\hline \multicolumn{3}{|c|}{$\begin{array}{l}\text { CUADRO 1. Perspectivas en la línea de investigación sobre el pensamiento del (a) profesor (a). } \\
\text { Fundamentada en Gallego (1991) }\end{array}$} \\
\hline NOMBRE & CARACTERISTICAS & AUTORES \\
\hline $\begin{array}{l}\text {-Toma de decisiones del (a) profesor } \\
\text { (a) durante la ensenanza interacti- } \\
\text { va }\end{array}$ & $\begin{array}{l}\text {-Ensenanza como activided técnice, altemente } \\
\text { cognitive } \\
\text {-Modelo racionalisla }\end{array}$ & $\begin{array}{l}\text { Peterson y Clark (1978) } \\
\text { Stem (1983) }\end{array}$ \\
\hline $\begin{array}{l}\text {-Modelos proceso ciclico- Mode- } \\
\text { los ciclico de pensamiento del (a) } \\
\text { profesor(a) } \\
\text {-Modelo del Razonamiento Peda- } \\
\text { gógico y Accion }\end{array}$ & $\begin{array}{l}\text {-Buscanintegrarpensamiento y acción } \\
\text {-Profundizar en el conocimiento de la practica del } \\
\text { (a) profesor (a) }\end{array}$ & $\begin{array}{l}\text { Anning (1987) } \\
\text { Shulman (1987) }\end{array}$ \\
\hline $\begin{array}{l}\text {-Estudio comparativo entre profe- } \\
\text { sores noveles y expertos } \\
\text {-Investigacion sobre planiticacion } \\
\text { del (a) profesor (a) }\end{array}$ & $\begin{array}{l}\text {-Frente a cuestionamientos de la investigacion } \\
\text { "psicologista",se aborda el probleme del curriculo } \\
\text { y el conocimiento de la materia en relación con la } \\
\text { ensenanza y el pensamiento del (a) profesor(a) }\end{array}$ & Clark(1985) \\
\hline $\begin{array}{l}\text {-Investigecion sobre pensamiento } \\
\text { y toma de decisiones de los pro- } \\
\text { fesores }\end{array}$ & $\begin{array}{l}\text {-Netureleza y contenido del pensamiento de los } \\
\text { profesores } \\
\text {-Influencia del conlexto organizativo y curricular } \\
\text {-Pensamientos profesoresconducta en clase } \\
\text {-Pensemientos profesor-pensemientos y conduc- } \\
\text { tas de los alumnos }\end{array}$ & $\begin{array}{l}\text { Lowyck (1990) } \\
\text { Pulman \& Duffy (1985) } \\
\text { Dillard (1987) } \\
\text { Calderheed (1988) }\end{array}$ \\
\hline
\end{tabular}

de investigación que predomina: uno, el enfoque dominante proceso-producto de la eficacia de la enseñanza y otro, el de procesos de pensamiento. Estos autores señalan que los investigadores del proceso-producto abordaron la relación entre la conducta de los alumnos en el aula y el rendimiento de los mismos; mientras que los investigadores sobre los procesos de pensamiento de los enseñantes se constituyeron en un enfoque paradigmático de la investigación (cuadro 2). De tal modo, que se indica que la introducción de la investigación sobre el pensamiento del (a) profesor (a) genera también un cambio en el paradigma de investigación. Antes anotamos que la introducción de esta línea

\section{CUADRO 2. Dominios de investigación propuestos por el Modelo de Pensamiento y de la acción del maestro} (elaborado con base en Clark y Peterson, 1997)

\begin{tabular}{|c|c|c|c|}
\hline $\begin{array}{c}\text { DOMINIO } \\
\text { INVESTIGADO }\end{array}$ & CARACTERISTICA & $\begin{array}{l}\text { EJEMPLO DE } \\
\text { AUTORES }\end{array}$ & $\begin{array}{c}\text { ALGUNOS } \\
\text { CUESTIONAMIENTOS }\end{array}$ \\
\hline $\begin{array}{l}\text { Dominio de ac- } \\
\text { ciondocente y sus } \\
\text { etectos observa- } \\
\text { bles }\end{array}$ & $\begin{array}{l}\text { Los investigadores del proceso pro- } \\
\text { ducto han supueslo generalmente } \\
\text { una causalidad unidireccional entre } \\
\text { la conducta de los docentes y la con- } \\
\text { ducta de los alumnos y el rendimien- } \\
\text { to }\end{array}$ & $\begin{array}{l}\text { Doyle (1977) } \\
\text { Dunkin y } \\
\text { Bidble(1974) }\end{array}$ & $\begin{array}{l}\text { No se han explorado relaciones reci- } \\
\text { procas sino unidireccionales entre la } \\
\text { conducta de los docentes y la } \\
\text { conducta de los alumnos y el rendi- } \\
\text { miento }\end{array}$ \\
\hline $\begin{array}{l}\text { Procesos de pensa- } \\
\text { miento de los do- } \\
\text { centes }\end{array}$ & $\begin{array}{l}\text { Comprende tres categonas; a) pla- } \\
\text { nincacion del docente (pensamien- } \\
\text { tos preactivos y postactivos); b) sus } \\
\text { pensamientos y decisiones interac- } \\
\text { tivos, y c) } \\
\text { sus teortas y creencias }\end{array}$ & $\begin{array}{l}\text { Jakcson } \\
(1968) \\
\text { Crist, Mant } \\
\text { y Peterson } \\
\text { (1974) }\end{array}$ & $\begin{array}{l}\text {-Dado que el proceso de ensenanza } \\
\text { es ciclico, la distincion entre pensa- } \\
\text { miento preactivos y postactivos es } \\
\text { imprecise } \\
\text {-Permanentemente se toman decisio- } \\
\text { nes y se hace cambios y no en unos } \\
\text { tiempos detemtinados. }\end{array}$ \\
\hline
\end{tabular}


de trabajo ha tenido implicaciones no solo de indole conceptual, sino además epistemológico.

Clark y Peterson (1997) realizan una revisión exhaustiva de las diferentes investigaciones; describen tanto los resultados como los aspectos metodológicos, entre otros, se destacan estudios sobre la planificación, los pensamientos y decisiones interactivas de los docentes, y las teorías implícitas de los mismos.

Estos autores resaltan el surgimiento de una nueva línea de investigación en la que se hace relevante el pensamiento del (a) profesor (a) tanto en la enseñanza como en el reconocimiento de este como profesional reflexivo. En particular, destacan que el docente como profesional formula explícitamente sus teorías y creencias, y aborda procesos de reflexión desde los cuales se convierte en investigador.

Destacan finalmente estos autores que la investigación en torno al pensamiento de los (as) profesores (as) ha permitido no sólo comprender estos procesos, sino además, ha dado luces frente a cómo se debe pensar sobre la enseñanza. Así, el surgimiento de esta línea de investigación ha posibilitado la comprensión del (a) profesor (a) como profesional, ha estado relacionada con cambios conceptuales y epistemológicos de la investigación en enseñanza, y además, ha posibilitado otras comprensiones sobre la misma.

Como señalamos, Gallego (1991) indica que se ha dado un cambio de la investigación sobre modelos en la toma de decisiones, hacia el pensamiento en acción y al estudio del conocimiento práctico del (a) profesor (a). En este sentido, cabe destacar que hemos aludido a textos de 1990 y 1991, pero aún en 1993, Gilroy planteó diferentes problemas para abordar el conocimiento del (a) profesor (a), lo hizo en relación con la construcción de un cuerpo de conocimientos que para algunos deben ser universalmente unificados, estos son: en la formación inicial de profesores se presenta la "incapacidad para construir un cuerpo unificado de conocimientos, a partir del cual hacer evolucionar sus prácticas educativas" (Roth, 1987); Una década más tarde, aún se indica la "lamentable ausencia de un cuerpo de conocimientos universalmente aceptado por los prácticos" (Watts, 1982); muchos profesores apoyan la idea de que la "experiencia es [...] lo que más cuenta" (Hargreaves, 1984); debe evitarse aplicar el término profesional a la enseñanza, porque "los profesores son ateóricos en el nivel de su enseñanza cotidiana" (Hoyle, 1974).

Problemas como los anteriores nos siguen planteando interrogantes frente a: ¿qué entendemos como conocimiento profesional? ¿Acaso para ello es necesario centrarnos en desarrollos teóricos que desconozcan la práctica? ¿Es claro que pretendemos que este sea de carácter universal? En este sentido, a continuación señalamos algunos planteamientos en relación con la didáctica de las ciencias como disciplina.

\section{El conocimiento profesional como línea de investigación en la didáctica de las ciencias}

Si retomamos el análisis realizado por Porlán (1998), es posible ubicar el reconocimiento de la línea de investigación del conocimiento profesional de los (as) profesores (as) desde la perspectiva de la didáctica de las ciencias como disciplina particular. Cabe destacar que en este proceso de desarrollo de la didáctica de las ciencias la crisis de la racionalidad científico-técnica provoca en esta disciplina redefiniciones teóricas y metodológicas hacia una concepción más relativa del conocimiento. Esta visión contextualizada de la ciencia pone en cuestionamiento muchos de los supuestos iniciales del currículo, desde los cuales se pretendía trasladar a la escuela la lógica de las disciplinas científicas (Porlán, 1998). Es desde esta consideración relativa del conocimiento que es posible considerar que en la escuela se producen otros conocimientos epistemológicamente diferenciados.

Aparece así el conocimiento profesional como una de las problemáticas que desde la didáctica se considera relevante. En el desarrollo de esta didáctica es posible reconocer una línea consolidada en torno al pensamiento de los alumnos, y una emergente, dadas las dificultades para conectar los resulta- 
dos de investigación con la actividad docente, en torno al conocimiento y la práctica de los (as) profesores (as), como indica Porlán (1998), citando a Martinand (1996) y Develay (1996). Pues resulta paradójico que incluso aquellos que cuestionaban el desconocer las concepciones de los estudiantes en el proceso de enseñanza, ignoraban las concepciones y los aspectos laborales y contextuales del docente (Porlán, 1998). Y es que el desarrollo profesional está en relación con el desarrollo de la misma disciplina "[...] una profesión científica debe ser contemplada como una "entidad histórica", o "población", cuyo desarrollo institucional es paralelo al desarrollo intelectual de la disciplina por el cual acepta la responsabilidad" (Toulmin, 1972, p. 268).

Desde esta perspectiva se asume que el o la profesor(a) elabora un conocimiento particular, como indica Hashweh (2005), por ejemplo, cuando un profesor planea para la enseñanza de un tópico específico, él o ella se plantean diferentes respuestas a preguntas que hacen alusión a aspectos como: nivel de detalle en que se aborda el tópico, posibles relaciones con otras ideas relevantes, dificultades de los estudiantes que se pueden presentar, representaciones (analogías, demostraciones, ejemplos, etc.) que se pueden usar, posibles procesos de evaluación.

En los últimos años varios autores resaltan la importancia de considerar el conocimiento profesional, tal como se expone a continuación: (Cuadro 3)

Así, el reconocer el conocimiento profesional de los profesores de ciencias como conocimientos particulares ha estado estrechamente articulado con el desarrollo de la didáctica de las ciencias como disciplina, en la que se resalta que hay problemas específicos que no han sido abordados por otra comunidad; que hay un objeto de estudio particular; una comunidad que construye teorías y que acepta la responsabilidad del desarrollo intelectual de esta disciplina. Y que también reconoce que frente al proceso de relativización del conocimiento se requiere del cuestionamiento de visiones absolutistas para aceptar que son posibles

\section{CUADR0 3. Algunos planteamientos que destacan al conocimiento de los (as) profesores (as) como un conocimiento diferenciado. (Elaborado con base en Martínez, 2000)}

\begin{tabular}{|l|c|}
\hline ASPECTOS MENCIONADOS & AUTOR \\
\hline $\begin{array}{l}\text { Reconocer el conocimiento de los profesores como un conocimiento particular diferencia- } \\
\text { do, relevante, es probablemente uno de los motivos fundamentales, para lo rarel desarro- } \\
\text { llo profesional. }\end{array}$ & $\begin{array}{c}\text { Furió } \\
\text { (1994) }\end{array}$ \\
\hline $\begin{array}{l}\text { Es necesano reconocer que los profesores tenemos ideas sobre la ensenanza y el aprendi- } \\
\text { zaje, pero también de favorecer la construccion de ese conocimiento fundamentado teo- } \\
\text { ricamente sobre lo que si ninca ensenar ciencias. }\end{array}$ & $\begin{array}{c}\text { Gil } \\
\text { (1991) }\end{array}$ \\
\hline $\begin{array}{l}\text { Los dedicados a la "practica de la educación deben poseer alguna "teoría" previa de la } \\
\text { educacion que estructure sus actividades y guíe sus decisiones". }\end{array}$ & $\begin{array}{c}\text { Carr y Kemmis } \\
\text { (1988) }\end{array}$ \\
\hline $\begin{array}{l}\text { El "conocimiento practico del profesor" es un conocimiento que le ermite actuar en una } \\
\text { situacion de accion }\end{array}$ & $\begin{array}{c}\text { Richardson } \\
(1990)\end{array}$ \\
\hline $\begin{array}{l}\text { Actuamos con base en nuestras ideas, el profesor desarrolla un modelo de la situacion y } \\
\text { actua con base en él. }\end{array}$ & $\begin{array}{c}\text { Pérez y Gimeno } \\
\text { (1988) }\end{array}$ \\
\hline $\begin{array}{l}\text { Los profesores usan un conocimiento en su practica “Conocimienlos Profesionales" que } \\
\text { estructuran su proceder. }\end{array}$ & $\begin{array}{c}\text { Bromme } \\
(1988)\end{array}$ \\
\hline $\begin{array}{l}\text { El profesor es un "sujeto epistemologico" que genera nuevas teon'as sobre su propia prác- } \\
\text { tica. }\end{array}$ & $\begin{array}{c}\text { Kroath } \\
(1989)\end{array}$ \\
\hline $\begin{array}{l}\text { Lo relevante no son las tareas en si mismas (comprender el contenido, adaptar el conteni- } \\
\text { do, etc.), sino las comprensiones parliculares de los profesores. }\end{array}$ & $\begin{array}{c}\text { Reynolds } \\
(1992)\end{array}$ \\
\hline
\end{tabular}


otras formas de conocimiento diferentes a las del conocimiento científico. Que en la escuela el conocimiento científico no es el único referente, y que allí se elaboran otros conocimientos producidos por actores particulares con formas de producción de validación también particulares.

Desde esta mirada se resalta el papel fundamental del (a) profesor (a), como profesional, en los procesos de enseñanza y en la generación de los cambios educativos. Como lo plantearon Clark y Peterson (1997), uno de los aspectos que pone de realce la investigación sobre el pensamiento del (a) profesor (a) es su relación con la comprensión y elaboración de propuestas de enseñanza. En este sentido se plantea que son los (as) profesores (as) quienes pueden hacer evolucionar los modelos de enseñanza, por lo que se hace necesario investigar sus concepciones (Porlán y Rivero, 1998).

Más aún, como lo señala Carr (1993), el proceso de "perfeccionamiento" de los (as) profesores (as) se basa en la revisión del conocimiento elaborado por ellos mismos, más que en aplicar el conocimiento producido por la investigación. De tal modo, que como lo indica este autor, es a través del reconocimiento de la posibilidad de saber y creación de los docentes, ${ }^{5}$ como se rechaza el autoritarismo externo y el papel pasivo del docente.

El desarrollo del conocimiento profesional de los (as) profesores (as) tiene implicaciones de carácter social, no sólo frente a una disciplina en la que se reconoce y asume como productor de conocimiento, sino además frente a las diferentes instituciones que definen políticas educativas, y frente a los diferentes mediadores desde los cuales el profesor ha de dejar de ser considerado como simple receptor y técnico que aplica los productos elaborados por otros. De esta manera, como lo afirma Carr (1993), se rechaza la perspectiva del (a) profesor (a) como técnico (a), y se reconocen sus desarrollos y obligaciones profesionales. Desde esta perspectiva, vale señalar que según Carr y Kemmis (1988), para que la enseñanza sea una actividad más genuina-

5 Fijémonos que Carr se refiere a maestras, denotando también un carácter de género. Parece que correspondió a los momentos iniciales de la profesión. Este sería un análisis propio para otro momento. mente profesional, es necesario que las actitudes y prácticas de los docentes se fundamenten más profundamente en la teoría e investigación educativa; que se amplíe la autonomía profesional de los maestros; y se generalicen sus responsabilidades profesionales.

De tal modo que desde esta identificación del (a) profesor (a) como profesional, se resaltan tres elementos centrales: la producción de un conocimiento particular, un conocimiento práctico fundamentado (en términos de Porlán, 1998), así como la autonomía y las responsabilidades profesionales en relación con la disciplina y con la sociedad.

Como lo indicamos antes, la literatura reporta diferentes investigaciones realizadas en la línea del conocimiento del (a) profesor (a), como una línea que apenas ha surgido en 1987 propuesta por Shulman, con base en la línea de investigación centrada en el pensamiento del (a) profesor (a), y del cual Shulman fue también creador. El enfoque sobre el pensamiento del (a) profesor (a) trata más de abordar las creencias y los juicios de los (as) profesores (as) desde un enfoque psicológico, centrado más en la investigación en los (as) profesores (as) de primaria; mientras que en la línea sobre el conocimiento del (a) profesor (a) destacan el papel de los contenidos escolares (López, 1995), también Perafán (2004) indica que se ha dado un cambio fundamental en esta línea de investigación, que pasa de abordar la pregunta acerca de qué piensan los (as) profesores (as) a qué, cómo, conocen los (as) profesores (as).

Todos estos autores coinciden en señalar que es necesario diferenciar epistemológicamente el conocimiento profesional de los (as) profesores (as). Justamente las propuestas actuales señalan como propósito en la formación inicial de los (as) profesores (as), la construcción de un conocimiento práctico profesional: "Una de las ideas que más potencialidad está teniendo en nuestro trabajo es la

6 En este sentido, habría que analizar en los contextos particulares, si estos aspectos son posibilitados y de qué manera, por ejemplo, ¿las consideraciones que hace la legislación educativa lo favorecen?, no sólo si indica que el profesor es un profesional, sino además, si las normas que se expiden son coherentes con este planteamiento. 
pretensión de que los futuros profesores construyan un conocimiento práctico profesional, no dirigido a la mera acción sino a la intervención intencionada y fundamentada" (Azcárate et ál., 1997, p. 1). En este sentido, es posible plantear una estrecha relación entre las propuestas de formación de profesores y el reconocimiento del conocimiento de los (as) profesores (as) como un conocimiento particular, tal como lo plantean Porlán y Rivero (1998).

Desde las investigaciones se ha empezado a explorar la naturaleza, forma, organización y contenido del conocimiento del (a) profesor (a), como indican Grossman et ál. (2005); al respecto se pueden mencionar los trabajos de Calderhead y Miler (1985) quienes aluden a un conocimiento de acción pertinente; Elbaz (1983), quien resalta que las elecciones instructivas del (a) profesor (a) se basan en su conocimiento de la materia, la comprensión de los estudiantes y el contexto de instrucción; Feiman-Nemser y Buchmann (1985) aluden a un "pensamiento didáctico"; McEwan (1987) a las "interpretaciones didácticas" de la materia; para estos autores, señalan Groosman et ál. (2005), parece que hay acuerdo con el planteamiento de Dewey según el cual "los profesores deben 'psicologizar' la materia para los estudiantes" (p. 7). Cabría preguntarse ¿qué se entiende por psicologizar, por integrar, transformar el contenido?, aspecto que no abordamos en este escrito, pero que consideramos fundamental para dar cuenta de las particularidades epistemológicas de este conocimiento.

En todo caso, hay coincidencia en señalar que existe una elaboración particular por parte del (a) profesor (a), que constituye uno de los desafíos de los (as) profesores (as) principiantes:

\section{CUADRO 4. Algunas propuestas sobre el Conocimiento Profesional del (a) profesor (a).} (Elaborado con base en van Driel, Verloop y de Vos, 1998)

\begin{tabular}{|c|c|c|}
\hline AUTORES & DENOMINACION & CATEGORIAS/COMPONENTES \\
\hline $\begin{array}{l}\text { Shulman } \\
(1986, \\
1987)\end{array}$ & $\begin{array}{l}\text { Conocrrmento de base para la ensenanza: Es el conoci- } \\
\text { miento de base ensenanza. }\end{array}$ & $\begin{array}{l}\text { Conocimiento del contenido, el Conocimiento } \\
\text { Pedagogico del para la Contenido, y el cono- } \\
\text { cimiento curricular, la pedagogia general. los } \\
\text { alumnos y sus caracteristicas, los contextos edu- } \\
\text { cativos y los lines educativos }\end{array}$ \\
\hline $\begin{array}{l}\text { Grossman } \\
(1990)\end{array}$ & $\begin{array}{l}\text { Conocimiento Pedagogico de Contenido (Pedagogical Con- } \\
\text { tent Knowledge): conjunto de estrategias y representacio- } \\
\text { nes de conocimiento para la enseñanza de temas específi- } \\
\text { cos y la comprensión del conocimiento de los estudianles. } \\
\text { las concepciones e ideas erréneas de estos temas }\end{array}$ & $\begin{array}{l}\text { El conocimiento de la materia, el conocimiento } \\
\text { pedagógico general y el conocimiento del con- } \\
\text { texto }\end{array}$ \\
\hline $\begin{array}{l}\text { Marks } \\
(1990)\end{array}$ & $\begin{array}{l}\text { Conocimiento Pedagogico de Contenido (Pedagogical Con- } \\
\text { tent Knowledge): un proceso integrador girando en torno a } \\
\text { la interpretación del conocimiento de la materia y la especi- } \\
\text { ficación del conocimiento pedagógico general }\end{array}$ & $\begin{array}{l}\text { Amplio el modelo de Shulman. incluyendo en el } \\
\text { CPC el conocimiento de la materia en si misma, } \\
\text { asi como el conocimiento de los medios de co- } \\
\text { municación para la instrucción. }\end{array}$ \\
\hline $\begin{array}{l}\text { Cochran, } \\
\text { DeRuiter, y } \\
\text { el Rey (1993). }\end{array}$ & $\begin{array}{l}\text { Conocer pedagógico de contenido (Pedagogical Content } \\
\text { Knowing): [C2]comprensi6n integrada de un maestro en tor- } \\
\text { no a los componentes }\end{array}$ & $\begin{array}{l}\text { Pedagogía, el contenido de la materia.las carac- } \\
\text { teristicas delos estudiantes, y el contexto am- } \\
\text { biental de aprendizaje }\end{array}$ \\
\hline $\begin{array}{l}\text { Fernández- } \\
\text { Balboa y } \\
\text { Stiehl (1995) }\end{array}$ & $\begin{array}{l}\text { Conocimiento Pedagógico de Contenido (Pedagogical Con- } \\
\text { tent Knowledge): integracion de los componentes de cono- } \\
\text { cimiento }\end{array}$ & $\begin{array}{l}\text { La materia, los estudiantes, las estrategias de ins- } \\
\text { trucción. el contexto de la ensenanza, y uno de } \\
\text { los propósitos de ensenanza. }\end{array}$ \\
\hline $\begin{array}{l}\text { Van Driel } \\
\text { Verloop, de } \\
\text { Vos(1998) }\end{array}$ & $\begin{array}{l}\text { Conocimiento Artesanal (Teachers' Craft Knowledge): Es un } \\
\text { conocimiento integrado que representa la sabiduría acu- } \\
\text { mulada de los docentes con respecto a su práctica docente } \\
\text { Comprende los tipos de conocimiento que realmente guian } \\
\text { el maestros durante la práctica de aula. }\end{array}$ & $\begin{array}{l}\text { El CPC como una forma específica de este cono- } \\
\text { cimiento artesanal (implica una transformación } \\
\text { del conocimiento dela materia) }\end{array}$ \\
\hline
\end{tabular}


"[...] uno de los primeros desafíos que enfrentan los profesores principiantes se refiere a las transformación de su conocimiento disciplinar en una forma de conocimiento que es apropiada para los estudiantes y específica para la tarea de enseñanza" (Grossman et ál., 2005, p. 20).

El dar cuenta de estas particularidades ha llevado al desarrollo de diferentes propuestas en torno al conocimiento del (a) profesor (a), como las que se señalan en el cuadro 4

\section{A modo de conclusión}

Es necesario precisar que los trabajos en torno al pensamiento del (a) profesor (a) permitieron reconocerlo como productor de conocimiento, y son la base fundamental para la línea en torno al cconocimiento profesional de los profesores. Tal como lo indica Porlán (1998), para el desarrollo de la didáctica de las ciencias fueron relevantes los aportes de la psicología, que también lo fueron en el desarrollo de esta línea de investigación. Vemos cómo nos referimos a un campo de investigación reciente, tomando como referencia la propuesta de Shulman (1987), que es señalada como el origen fundamental, y que apenas tiene 30 años de desarrollo.

Aunque no ha sido motivo de este trabajo, en el contexto de esta línea de investigación se han adelantado investigaciones que permiten adentrarse en propuestas más específicas en torno al conocimiento didáctico de disciplinas particulares (Cochran y Jones, 1998), por ejemplo, en el conocimiento didáctico de la Química (Martín, 1994; van Driel, 2002), y en el conocimiento didáctico de la Biología (Valbuena, 2007), de la Física (Sperandeomineo et ál., 2005). De igual modo, es pertinente señalar que esta línea ha estado relacionada con el reconocimiento de que en la escuela se produce un conocimiento particular, este es, el conocimiento escolar (García, 1998; Martínez, 2000). Los desarrollos se han dado al punto de que el Internacional Handbook of Science Education (Fraser y Tobin, 1998) incluye una sesión completa dedicada a la formación del (a) profesor (a) en la que se abordan artículos referidos a su conocimiento; también en Perales y Cañal (2000) se abordan en la formación de profesores aspectos relacionados con el conocimiento del mismo, y más recientemente, el Handbook of Research on Science Education aborda en la parte IV referida a la Formación de profesores varios capítulos en torno al conocimiento del (a) profesor (a) (por ejemplo Abell, 2007).

No lo tratamos aquí, pero diferentes trabajos aportan en la comprensión y diferenciación de componentes y referentes del conocimiento profesional de los profesores, así como de la organización y construcción de este conocimiento. Sin embargo, cabe preguntarnos, en nuestros contextos particulares, ¿cuáles son las características del conocimiento profesional de los profesores de las diferentes áreas de conocimiento tales como la de los (as) profesores (as) de Química, de Artes, de Física o de Tecnología entre otros?; pero también, de aquellos (as) profesores (as) que no han sido formados como docentes, es decir, los ingenieros que se asumen como profesores, los médicos que forman nuevos médicos, etc. En este sentido, es necesario indagar para comprender esas maneras particulares que los (as) profesores (as) desarrollan, y que se pueden transformar en un conocimiento más público (Hashweh, 2005); e igualmente investigar cómo algunas personas aprenden los diversos tipos de reflexión en acción (Schön, 1996). Pues las diferentes investigaciones nos permiten señalar que el profesor elabora un conocimiento particular cuando enseña; así por ejemplo, frente a la enseñanza de un tópico particular se pregunta respecto a consideraciones propias de su profesión, tales como el nivel de detalle que se pretende para unos estudiantes determinados; las posibles relaciones con otras ideas; las posibles dificultades de los estudiantes y alternativas para superarlas; las concepciones previas de los estudiantes, etc. (Hashweh, 2005).

Como lo señalan Cochran y Jones, (1998), necesitamos un trabajo teórico para avanzar la hipótesis acerca de las dimensiones en las cuales el conocimiento didáctico del contenido debe ser diferente del conocimiento del contenido o del conocimiento pedagógico, en términos de su desarrollo, estruc- 
tura, contenido y uso; pues aunque Shulman dio origen a Pedagogical Content Knowlegde ${ }^{7}$ (PCK), los investigadores le han dado diferentes interpretaciones y significados (Park y Oliver, 2007). De tal manera que se podría decir que asistimos a un momento fructifero del conocimiento, ya no solamente de la didáctica como campo de investigación en general, sino en particular, en torno a la línea de investigación sobre el conocimiento profesional de los profesores de ciencias (Martínez, 2000, 2003).

\section{Agradecimientos}

Agradezco las observaciones, sugerencias y valiosos aportes realizados por el doctor Edgar Valbuena y la maestra Vilma Martínez Rivera. Toda imprecisión o falencia en el texto son de mi exclusiva responsabilidad.

\section{Referencias}

Abell, S. (2008). Twenty Years Later: Does pedagogical content knowledge remain a useful. International Journal of Science Education, 30 (10), 1405-1416.

Abell, S. (2007). Research on Science teacher Knowledge. En Abell, S. and Lederman, N. Handbook of research on science education (pp. 1105-1149). London: Lawrence Erlbaum Associates.

Aning, A. (1987). An analysis of six primary teachers' understanding of how children learn and its relationship to their teaching strategies (Paper presented at Conference on Teachers' Professional Learning, University of Lancaster, July).

Azcárate, P., R. Martín y R. Porlán (1997). Una perspectiva epistemológica para analizar y transformar la formación inicial del profeso-

7 En el habla hispana suele traducirse como "conocimiento didáctico del contenido", aspecto relevante por analizar, dado que evoca las diferencias entre pedagogía y didáctica, (Sugiero eliminar esta frase pues el artículo prácticamente finalizó)

REVISTA CIENTÍFICA / ENERO -DICIEMBRE DE 2009 / No. 11 / BOGOTÁ, D.C. rado (Comunicación presentada en el Congreso Enseñanza de las Ciencias, Murcia).

Bromme, R. (1988). Conocimientos profesionales de los profesores. Enseñanza de las Ciencias, $6(1), 19-29$.

Calderhead, J. (1988). Conceptualización e investigación del conocimiento profesional de los profesores. En L. M. Villar (ed.). Conocimiento, creencias y teorías de los profesores. Murcia: Marfil.

Carr, W. (1993). Calidad de la Enseñanza e Investigación-Acción. Sevilla: Díada Editora.

Carr, W. y S. Kemmis (1988). Teoría crítica de la enseñanza. La Investigación en la acción en la formación del profesorado. Barcelona: Martínez Roca.

Claderhead, J. y E. Miller (1985, September). The integration of subject matter knowledge in student teachers'classroom practice (Paper presented at the annual meeting of the British Educational Research Association, Sheffield).

Clark, C. (1985). Ten Years of Conceptual Development in Research on Teachers Thinking (Paper presented to the Conference of the ISATT, Tilburg, The Netherlands, May 28).

Clark, C. y P. Peterson, (1997). Procesos de pensamiento de los docentes. En: Wittrock (comp.). La investigación de la enseñanza, III. Profesores y alumnos. Barcelona: Ministerio de Educación (Título original de 1990, Handbook of Research on Teaching).

Cochran, K. and L. Jones (1998). The subjetc matter knowledge of preservice science teachers. En: Fraser and Tobin (eds.). International Handbook of Science Education. London: Kluwer Academic Publishers.

Cochran, K. F., J. A. Deruiter \& R. A. King (1993). Pedagogical content knowing: An integrative 
model for teacher preparation. Journal of Teacher Education, 44, 263-272.

Crist, Marx y Peterson (1974). Teacher behavior in the organizational domain (Informe presentado al National Institute of Education). Stanford, CA: Stanford Center for R\&D in Teaching.

Develay, M. (1996). Didactique et perspectives de formation. Educations, enero, febrero, 4952 .

Dewey, J. (1983). The child and the curriculum. In: J. A. Boydston (ed.). Jonh Dewey: The middle works, 1899-1924. Vol. 2: 1902-1903 (pp. 273-91). Carbondale, IL: Southern Illinois University Press.

Dillard, J. (1987). Getting into the bargain: A study of Interactional Thinking and Improvisation (Paper presented at annual meeting of the A.E.R.A., Washington, April).

Doyle (1977). Paradigms for research on teacher effectiveness. Review of Research in Education, 5, 163-198.

Dunkin y Bidble (1974). The study of teaching. New York: Holt, Rinehart\& Winston.

Elbaz, F. (1983). Teacher thinking: A study of practical knowledge. New York: Nichols.

Fraser, B. y K. Tobin (eds.) (1998). International handbook of science education. London: Kluwer Academic Publishers.

Feiman-Nemser, S. (1985a). The First year of teacher preparation: transition to pedagogical thinking (Research Series No 156). East Lansing: Michigan State University, Institute for Research on Teaching.

Fernández-Balboa, J.-M. \& J. Stiehl (1995). The generic nature of pedagogical content knowledge among college professors. Teaching \& Teacher Education, 11, 293-306.
Furió, C. (1994). Tendencias actuales en la formación del profesorado de ciencias. Enseñanza de las Ciencias, 12 (2), 188-199.

García Díaz, J. E. (1998). Hacia una teoría alternativa sobre los contenidos escolares. Sevilla: Díada.

Gallego, M. (1991). Investigación sobre pensamientos del profesor: aproximaciones al estudio de las "Teorías y creencias de los profesores". Revista española de pedagogía, año XLIX (189), 287-346.

Gil, D. (1991). ¿Qué hemos de saber y saber hacer los profesores de ciencias? (Intento de síntesis de las aportaciones de la investigación didáctica). Enseñanza de las Ciencias, 9 (1), 69-77.

Gil, D., J. Carrascosa y F. Martínez (2000). Una disciplina emergente y un campo específico de investigación. En: J. Perales y P. Cañal. Didáctica de las ciencias experimentales. Alcoy: Marfil.

Gilroy, P. (1993). El conocimiento profesional y el profesor principiante. En: W. Carr. La calidad de la enseñanza e investigación acción. Sevilla: Díada Editora.

Grossman, P., S. Wilson, L. Shulman (2005). Profesores de sustancia: el conocimiento de la materia para la enseñanza. Profesorado. Revista de currículum y formación del profesorado, (9) 2, 1-24.

Grossman, P., S. Wilson y L. Shulman (2005). Profesores De Sustancia: El conocimiento de la materia para la enseñanza. Profesorado. Revista de currículum y formación del profesorado, 9 (2), 1-24

Grossman, P. L. (1990). The making of a teacher: Teacher knowledge and teacher education. New York: Teachers College Press.

Grundy, S. (1993). Más allá de la profesionalidad. En: W. Carr. La calidad de la enseñanza e investigación acción. Sevilla: Díada Editora. 
Hashweh, M. (1996). Effects of science teacher's epistemological beliefs in teaching. Journal of Research in Science Teaching, 33 (1).

Hashweh, M. (2005). Teacher pedagogical constructions: a reconfiguration of pedagogical content knowledge. Teachers and Teaching: theory and practice, 11 (3), 273-292.

Hargreaves (1984). Experience Counts, Theory doesn't: How teacher talk about their work". Sociology of education, 57, 244-254

Hoyle (1974). Professionality, professionalism and control in teaching. London Educational Review, 3 (2), 13-19.

Jackson (1968). Life in classrooms. Nueva York: Holt, Rinehart \& Winston (trad. al castellano: La vida en las aulas. Madrid: Morata, 1975).

Kroath, F. (1989). How do Teachers Change their Practical theories? Cambridge Journal of Education, 19 (1), 55-64.

López, I. (1995). El conocimiento profesional de los profesores acerca de las concepciones de los alumnos. Dos estudios de caso en la enseñanza y el aprendizaje de las ciencias. Tesis doctoral inédita. Universidad de Sevilla.

Lowyck, J. (1990). A Process Análisis of Teaching. Report 21. Document ERIC.Ed. 190513.

Marks, R. (1990). Pedagogical content knowledge: From a mathematical case to a modified conception. Journal of Teacher Education, 41, 3-11.

Martín, R. (1994). El conocimiento del cambio químico en la formación inicial del profesorado. Estudio de las concepciones disciplinares y didácticas de los estudiantes de magisterio. Tesis doctoral inédita. Universidad de Sevilla.

Martinand, J. (1996). Dóú est venue la didactique? Educations, enero, febrero, 22-25.
Martínez, C. (2000). Las propuestas curriculares sobre el conocimiento escolar en el área de conocimiento del medio: dos estudios de caso en profesores de primaria. Tesis Doctoral. Programa Didáctica de las Ciencias Experimentales y Sociales, un enfoque interdisciplinar. Universidad de Sevilla.

Martínez, C. (2003). Conocimiento profesional de los profesores de ciencias: retos contemporáneos. Tecne Episteme y Didaxis (Extra), 42-51.

Martínez, C. (2008). El conocimiento profesional de los profesores de ciencias CPPC. Desarrollos de la línea de investigación. Seminario doctoral "El conocimiento profesional de los profesores de ciencias". Doctorado Interinstitucional en Educación. Universidad Distrital Francisco José de Caldas.

Mcewan, H. (1987). Interpreting the subject domains for students: Towards a rhetorical theory of teaching. Unpublished doctoral dissertation. Seatle: University of Washington.

Molina, A. (2006). Conversaciones personales. Bogotá: Universidad Distrital Francisco José de Caldas.

Munby y Rusell (1998). Epistemology and context in research on learning to teach science. En: Fraser and Tobin (eds.). International Handbook of Science Education. London: Kluwer Academic Publishers.

Nacional Institute of Education (1975). Teaching as clinical information processing (Informe del Panel 6, National Conference on Studies in Teaching). Washington, DC: National Institute of Education.

Park \& Oliver (2007). Revisiting the Conceptualisation of Pedagogical Content Knowledge (PCK): PCK as a Conceptual Tool to Understand Teachers as Professionals. Res Sci Educ \# Springer Science + Business Media B.V. 
Perafán (2004). La epistemología del profesor sobre su propio conocimiento profesional. Bogotá: Universidad Pedagógica Nacional.

Perales, F. y P. Cañal (comps.) (2000). Didáctica de las ciencias experimentales. Alcoy: Marfil.

Pérez, A. y J. Gimeno (1988). Pensamiento y acción en el profesor: de los estudios sobre la planificación al pensamiento práctico. Infancia y aprendizaje, 42, 37-63.

Peterson y Clarck (1978). Teacher's reports of their cognitive processes during teaching. American Educational Research Journal, 15, 555-565.

Porlán, R. (1998). Pasado, presente y futuro de la didáctica de las ciencias. Enseñanza de las Ciencias, 16 (1), 175-185.

Porlán, R. y A. Rivero (1998). El conocimiento de los profesores. Sevilla: Díada Editora.

Porlan, R., R. Martin, J. Martin, A. Rivero (1995). Evaluación formativa de un proyecto institucional de formación permanente del profesorado: El programa de actualización científica $y$ didáctica (Modalidad A). Memoria de investigación inédita. Universidad de Sevilla.

Putnan, J. \& G. Duffy (1985). The subtleties and complexities of instructional explanation in reading: A case study o fan expert (I.R.T.,

Richardson, V. (1990). Significant and Worthwhile Change in Teaching Practice. Educational Research (Oct.), 10-18.

Roth (1987). Towards a Delineation of Professional Knowledge. Kappa Delta Pi Record, 7 (1), 9-11.
Shavelson, R. y P. Stern (1983). Investigación sobre el pensamiento del profesor, sus juicios, decisiones y conducta. En: Gimeno y Pérez (eds.).

Schön, D. (1996). La crisis del conocimiento profesional y la búsqueda de una epistemología de la práctica. En: M. Pakman (comp.). Construcciones de la Experiencia humana. Vol. 1. Barcelona: Gedisa.

Shulman, L. S. (1986). Those who understand: knowledge growth in teaching, Educational Researcher, 15 (2), 4-14. (Trad. y edición española: El saber y entender de la profesión docente. En: Estudios Públicos. Chile: Centro de Estudios Públicos, 99, 2005, 195-224.

Shulman, L. S. (1987). Knowledge and Teaching. Foundations of the New Reform. Harvard Educational Review, 57 (1). (Trad. al español 2001. Conocimiento y enseñanza. Estudios públicos, 63, 163-196.

Shulman, L. S. (2001). Conocimiento y enseñanza. Estudios públicos, 63, 163-196. Traducción de L. S. Shulman (1987). Knowledge and teaching: Foundations of the new reform. Harvard Educational Review, 57, 1-22.

Shulman, L. (1989) Paradigmas y programas de investigación en el estudio de la enseñanza: una perspectiva contemporánea, I. Profesores y alumnos. Barcelona: Ministerio de Educación. (Título original de 1989, Handbook of Research on Teaching).

Sperandeo-Mineo, Fazio and Tarantino (2005). Pedagogical Content Knowledge Development and Pre-Service Physics, Teacher Education: A Case Study. Research in Science Education, 36 (3), 235-268.
Torres, R. (1994). Prólogo. En: P. Freire (2002). Cartas a quien pretende enseñar. 8 edición. México: Siglo Veintiuno editores.

Toulmin, S. (1972). Human Understanding. Vol. I: The collective use and evolution of concepts. 
Princeton University Press. (Trad. al español: La comprensión humana. Vol. I: El uso colectivo y la evolución de los conceptos. Madrid: Alianza Editorial, 1977).

Travers (ed.) (1973). Second Handbook of research on teaching. Chicago: Rand Mcnally.

Watts (1982). Can Campus-Based preservice education survive? Journal of Teacher Education, 33 (2), 3-41.

Valbuena, E. (2007). El conocimiento didáctico del contenido Biológico. Estudio de las concepcio- nes disciplinares y didácticas de futuros docentes de la Universidad Pedagógica Nacional (Colombia). Madrid: Universidad Complutense.

van Driel, J. H., N. Verloop, W. De Vos (1998). Developing Science Teachers' Pedagogical Content Knowledge. Journal of Research In Science Teaching, 35 (6), 673-695.

van Driel, De Jong, Verloop (2002). The development of preservice chemistry teachers pedagogical content knowledge. Science Education, 86 (4), 572550 